PROCEEDINGS OF THE

AMERICAN MATHEMATICAL SOCIETY

Volume 126, Number 10, October 1998, Pages 3083-3087

S $0002-9939(98) 04399-8$

\title{
THE FUNDAMENTAL GROUP OF A COMPACT METRIC SPACE
}

\author{
JANUSZ PAWLIKOWSKI
}

(Communicated by Andreas R. Blass)

\begin{abstract}
We give a forcing free proof of a conjecture of Mycielski that the fundamental group of a connected locally connected compact metric space is either finitely generated or has the power of the continuum.
\end{abstract}

Shelah [S], using models, absoluteness and Cohen's forcing method, gives a proof of the following conjecture of Mycielski.

Theorem. Suppose that $X$ is a compact metric space, which is connected and locally connected. Then the fundamental group of $X$ is either finitely generated or has the power of the continuum.

Thus in particular the group of rationals can't be the fundamental group of a connected locally connected compact metric space.

We can't drop 'compact' in the theorem - any countably generated group can be realized as the fundamental group of a Polish space, see [Sp]. We also can't drop 'locally connected' - the fundamental group of the 'tail of the peacock' is free with a countable infinity of generators. It seems to be open, however, whether a finitely generated group can be realized as the fundamental group of a compact metric space.

We present a forcing free proof of Mycielski's conjecture.

Definitions. Let $X$ be a metric space. A path from $x_{0}$ to $x_{1}$ inside $V \subseteq X$ is a continuous function $f:[0,1] \mapsto V$ with $f(0)=x_{0}$ and $f(1)=x_{1}$. $f$ is a loop at $x$ if $f(0)=f(1)=x$. The reversal of $f$, denoted by $f^{-1}$, is a path from $f(1)$ to $f(0)$ defined by $f^{-1}(t)=f(1-t)$. The diameter of $f$ is the diameter of the set $\{f(t): t \in[0,1]\}$.

A path $f$ is homotopic to another path $g, f \sim g$, if there is a homotopy from $f$ to $g$, i.e., a continuous function $F:[0,1] \times[0,1] \mapsto X$ such that $F(t, 0)=f(t)$, $F(t, 1)=g(t), F(0, s)=f(0)=g(0)$ and $F(1, s)=f(1)=g(1)$ (thus endpoints are kept constant; this is usually called 'a homotopy relative to $\{0,1\}$ '). If $f$ is a path from $x_{0}$ to $x_{1}$ and $g$ a path from $x_{1}$ to $x_{2}$, then $f * g$ is the concatenation of $f$ and $g$, i.e., a path from $x_{0}$ to $x_{2}$ defined by $(f * g)(t)=f(2 t)$ for $t \in[0,1 / 2]$ and $(f * g)(t)=$

Received by the editors August 17, 1996 and, in revised form, February 26, 1997.

1991 Mathematics Subject Classification. Primary 03E15, 55Q05; Secondary 04A20, 55Q52.

Key words and phrases. Baire category, fundamental group, perfect set.

The author was partially supported by KBN grant 2 P03A 01109 . The author thanks J. Mycielski for introducing him to [S].

(C)1998 American Mathematical Society 
$g(2 t-1)$ for $t \in[1 / 2,1]$. The iterated concatenation $f_{0} *\left(f_{1} *\left(\ldots\left(f_{n-1} * f_{n}\right) \ldots\right)\right)$ is written as $f_{0} * \cdots * f_{n}$.

The relation $\sim$ is an equivalence relation. If $X$ is path connected, the set of equivalence classes of loops at a given point $x \in X$, with multiplication and inverse defined from $*$ and ${ }^{-1}$, is a group called the fundamental group of $X$. This group doesn't depend on the choice of $x$.

Let $\mathbb{N}$ be the set of nonnegative integers. Let $x \in X$ and suppose that $f_{n}(n \in \mathbb{N})$ are loops at $x$ whose diameters converge to 0 . Then $\left(f_{0} * f_{1} * \cdots\right)$ is the pointwise limit of $f_{0} * \cdots * f_{n}$. The limit exists and is continuous, so it is a loop at $x$. In fact, if $t \in\left[1-2^{-n}, 1-2^{-(n+1)}\right]$, then

$$
\left(f_{0} * f_{1} * \cdots\right)(t)=f_{n}\left(\left(t-\left(1-2^{-n}\right)\right) \cdot 2^{n+1}\right) .
$$

Note also that

$$
\left(f_{0} * f_{1} * \cdots\right)=\left(f_{0} * \cdots * f_{n}\right) *\left(f_{n+1} * \cdots\right) .
$$

From now on we assume that $X$ is a connected locally connected compact metric space. Then $X$ is also path connected and locally path connected (a theorem of Mazurkiewicz, see $[\mathrm{K}]$ ).

Lemma 1. Suppose that the fundamental group of $X$ is not finitely generated. Then there exists $x \in X$ such that for each $n \in \mathbb{N}$ there exists a loop $f_{n}$ at $x$ which is of diameter $<2^{-n}$ and which is not homotopic to the constant loop at $x$.

Proof. Suppose otherwise. Then for each $x \in X$ there exists $n(x) \in \mathbb{N}$ such that every loop at $x$ which has diameter $<2^{-n(x)}$ is homotopic to the constant loop at $x$. By compactness there exists a cover of $X$ by path connected sets $V_{i}(i<k)$ and there exist points $x_{i} \in V_{i}(i<k)$ such that for every $i$ the diameter of $V_{i}$ is $<2^{-\left(n\left(x_{i}\right)+1\right)}$ and any loop at $x_{i}$ which has diameter $<2^{-n\left(x_{i}\right)}$ is homotopic to the constant loop at $x_{i}$.

Fix a path $g_{i}$ from $x_{0}$ to $x_{i}(i<k) ; g_{0}=$ the constant loop at $x_{0}$. For $i$ and $j$ such that $V_{i} \cap V_{j} \neq \emptyset$ fix a path $h_{i j}$ in $V_{i} \cup V_{j}$ going from $x_{i}$ to $x_{j}$. Note that any path $s$ from $x_{i}$ to $x_{j}$ which is contained in $V_{i} \cup V_{j}$ is homotopic to $h_{i j}$. Indeed, suppose that $n\left(x_{i}\right) \leq n\left(x_{j}\right)$. Then $s * h_{i j}^{-1}$ is a loop at $x_{i}$ and has diameter $<2^{-n\left(x_{i}\right)}$, so $s * h_{i j}^{-1}$ is homotopic to the constant loop at $x_{i}$. An elementary manipulation of this homotopy gives a homotopy from $s$ to $h_{i j}$. If $n\left(x_{i}\right)>n\left(x_{j}\right)$, consider $h_{i j}^{-1} * s$, a loop at $x_{j}$.

We shall show that the fundamental group of $X$ is generated by the (homotopy classes of) loops $\widetilde{h}_{i j}=g_{i} * h_{i j} * g_{j}^{-1}$. To this end, suppose that a loop $s$ at $x_{0}$ is given. By a change of scale $s \sim s_{0} * \cdots * s_{l}$, where each $s_{i}$ is a path inside one piece of our cover. Say $s_{i}$ goes from $y_{i}$ to $y_{i+1}$ inside $V_{\phi(i)} ; y_{0}=y_{l+1}=x_{0}, \phi(0)=\phi(l)=0$. For $i=0, \ldots, l$, fix inside $V_{\phi(i)}$ a path $t_{i}$ from $y_{i+1}$ to $x_{\phi(i)} ; t_{0}=s_{0}^{-1}, t_{l}=$ the constant loop at $x_{0}$. Let $\widetilde{s}_{i}=t_{i-1}^{-1} * s_{i} * t_{i}(i=1, \ldots, l)$. Clearly

$$
\widetilde{s}_{1} * \cdots * \widetilde{s}_{l} \sim s .
$$

Also, each $\widetilde{s}_{i}$, being a path from $x_{\phi(i-1)}$ to $x_{\phi(i)}$ inside $V_{\phi(i-1)} \cup V_{\phi(i)}$, must be homotopic to $h_{\phi(i-1) \phi(i)}$. Thus

$$
s \sim h_{\phi(0) \phi(1)} * \cdots * h_{\phi(l-1) \phi(l)},
$$

and hence also

$$
s \sim \widetilde{h}_{\phi(0) \phi(1)} * \cdots * \widetilde{h}_{\phi(l-1) \phi(l)} .
$$


For the sequel suppose that the fundamental group of $X$ is not finitely generated and let $x$ and $f_{n}(n \in \mathbb{N})$ be as claimed by Lemma 1. We shall find a set of size of the continuum of mutually non-homotopic loops. For $\alpha \in\{0,1\}^{\mathbb{N}}$ let $f_{n}^{\alpha}=$ the constant loop at $x$ if $\alpha(n)=0$, and let $f_{n}^{\alpha}=f_{n}$ otherwise. Define a loop $f_{\alpha}$ at $x$ as $\left(f_{0}^{\alpha} * f_{1}^{\alpha} * \cdots\right)$. Write $\alpha \approx \beta$ if $f_{\alpha} \sim f_{\beta}$. Then $\approx$ is an equivalence relation in $\{0,1\}^{\mathbb{N}}$. It is enough to prove that $\approx$ has continuum many equivalence classes.

Lemma 2. Suppose that $\alpha$ and $\beta$ from $\{0,1\}^{\mathbb{N}}$ differ exactly at one point. Then $\alpha \not \approx \beta$.

Proof. Suppose that $f_{\alpha} \sim f_{\beta}$. Let $n$ be the unique point at which $\alpha$ and $\beta$ are different. Then, for $m \neq n$ we have $f_{m}^{\alpha}=f_{m}^{\beta}$, hence $\left(f_{0}^{\alpha} * \cdots * f_{n-1}^{\alpha}\right)=\left(f_{0}^{\beta} * \cdots * f_{n-1}^{\beta}\right)$ and $\left(f_{n+1}^{\alpha} * \cdots\right)=\left(f_{n+1}^{\beta} * \cdots\right)$. Thus from

$$
\left(f_{0}^{\alpha} * \cdots * f_{n-1}^{\alpha} * f_{n}^{\alpha} * f_{n+1}^{\alpha} * \cdots\right) \sim\left(f_{0}^{\beta} * \cdots * f_{n-1}^{\beta} * f_{n}^{\beta} * f_{n+1}^{\beta} * \cdots\right),
$$

we get $f_{n}^{\alpha} \sim f_{n}^{\beta}$, which is a contradiction.

More definitions. We recall some basic facts about Polish spaces (see $[\mathrm{K}]$ ). A Polish space is a completely metrizable separable space. Let $Y$ be a Polish space. For a subset $A$ of $Y: A$ is nowhere dense if its closure has empty interior, $A$ is meager if it is a countable union of nowheredense sets, $A$ is comeager in an open set $U$ if $U \backslash A$ is meager, $A$ has the Baire property if its symmetric difference with some open set is meager. A nonmeager set with the Baire property is comeager in some nonempty open set. The Baire category theorem implies that a set which is comeager in a nonempty open set is nonmeager. The Kuratowski-Ulam theorem implies that if $A \subseteq Y \times Z$ is comeager in $U \times V$, where $U$ and $V$ are open subsets of Polish spaces $Y$ and $Z$, then

$$
\{y \in U:\{z \in V:\langle y, z\rangle \in A\} \text { is comeager in } V\}
$$

is comeager in $U$.

A subset $A$ of $Y$ is analytic if there exist a Polish space $Z$ and a closed set $D \subseteq Y \times Z$ such that $A$ is the projection of $D$ into $Y$. Analytic sets have the Baire property. Continuous preimages of analytic sets are analytic.

A subset $P$ of $Y$ is perfect if it is closed, nonempty, and has no isolated points. Perfect sets have the power of the continuum.

The set $\{0,1\}^{\mathbb{N}}$ becomes a Polish space (homeomorphic to the Cantor discontinuum) when viewed as the product of countably many copies of the two-point discrete space $\{0,1\}$. The canonical basis of $\{0,1\}^{\mathbb{N}}$ is the collection of all sets $[\sigma]=\{\alpha: \sigma \subseteq \alpha\}$, where $\sigma$ is a finite zero-one sequence, i.e., $\sigma:\{0,1, \ldots, n-1\} \mapsto$ $\{0,1\}$ for some $n$.

Lemma 3. $\approx$ has the Baire property as a subset of $\{0,1\}^{\mathbb{N}} \times\{0,1\}^{\mathbb{N}}$.

Proof. Let $H$ and $\mathbb{H}$ be respectively the spaces of all loops at $x$ and all homotopies between them, endowed with the sup metric. Both spaces are Polish. The homotopy relation $\sim$ restricted to $H$ is an analytic subset of $H \times H$. Indeed, it is the projection onto $H \times H$ of

$$
\{\langle\langle f, g\rangle, F\rangle: F \text { is a homotopy from } f \text { to } g\},
$$


which is a closed subset of $(H \times H) \times \mathbb{H}$. Note also that the function from $\{0,1\}^{\mathbb{N}}$ to $H$ which takes $\alpha$ to $f_{\alpha}$ is continuous. It follows that $\approx$ is analytic (as a continuous preimage of an analytic set), and thus has the Baire property.

Lemma 4. If $E \subseteq\{0,1\}^{\mathbb{N}} \times\{0,1\}^{\mathbb{N}}$ is an equivalence relation which has the Baire property and if $\neg x E y$ whenever $x$ and $y$ differ by one coordinate only, then $E$ is meager.

Proof. Should $E$ be nonmeager, it would be comeager in some basic neighbourhood $[\sigma] \times[\tau]$. By the Kuratowski-Ulam theorem,

$$
A=\{\alpha \in[\sigma]:\{\beta \in[\tau]: \alpha E \beta\} \text { is comeager in }[\tau]\}
$$

is comeager in $[\sigma]$. Let $n>$ the length of $\sigma$. Consider a map $\Phi:[\sigma] \mapsto[\sigma]$ defined by $\Phi(\alpha)(n)=1-\alpha(n)$ and $\Phi(\alpha)(i)=\alpha(i)$ for $i \neq n$. $\Phi$ is a homeomorphism and thus $\Phi[A]$ is comeager in $[\sigma]$. Choose $\alpha \in A \cap \Phi[A]$ and let $\gamma=\Phi(\alpha)$. Then $\alpha$ and $\gamma$ differ only at $n$, hence $\neg \alpha E \gamma$. Also, by the definition of $A$, we have in $[\tau]$ comeagerly many $\beta$ with $\alpha E \beta$. As $\gamma \in A$, the same is true about $\gamma$. Thus there exists $\beta$ with $\alpha E \beta$ and $\gamma E \beta$. But then $\alpha E \gamma$, which is a contradiction.

Remark. Another way to see that $E$ is meager might be as follows. Suppose for contradiction that $E$ is nonmeager. Consider $G=\{0,1\}^{\mathbb{N}} \times\{0,1\}^{\mathbb{N}}$ as a Polish group with coordinatewise addition mod 2. By the Baire category version of a theorem of Steinhaus (see $[\mathrm{O}]$ ), if $B \subseteq G$ has the Baire property and is nonmeager, then the difference set $B-B=\left\{b_{0}-b_{1}: b_{0}, b_{1} \in B\right\}$ contains a neighbourhood of the unit element $\langle\overline{0}, \overline{0}\rangle$ (here $\overline{0}=\langle 0,0, \ldots\rangle$ ). So, for each $\langle\delta, \epsilon\rangle \in G$, which is close enough to $\langle\overline{0}, \overline{0}\rangle$, there exist $\langle\alpha, \beta\rangle \in B$ such that $\langle\alpha+\gamma, \beta+\delta\rangle \in B$. For $n \in \mathbb{N}$ let $\epsilon_{n} \in\{0,1\}^{\mathbb{N}}$ be the function that takes value 1 at $n$ and 0 elsewhere. Then $\left\langle\epsilon_{n}, \overline{0}\right\rangle \longrightarrow\langle\overline{0}, \overline{0}\rangle$, when $n \longrightarrow \infty$. So, for large enough $n$ there exists $\langle\alpha, \beta\rangle \in B$ with $\left\langle\alpha+\epsilon_{n}, \beta\right\rangle \in B$. Applied to $B=E$ this yields that for large enough $n$ there exist $\alpha$ and $\beta$ such that $\alpha E \beta$ and $\alpha+\epsilon_{n} E \beta$, whence $\alpha E \alpha+\epsilon_{n}$. This contradicts Lemma 2.

Similar arguments show that if $E$ is Lebesgue measurable then it must be null.

Corollary. $\approx$ is meager.

Recall now the following theorem of Mycielski [M].

Theorem (Mycielski). Suppose that $Y$ is a Polish space without isolated points and that $R \subseteq Y \times Y$ is meager. Then there exists a perfect set $P \subseteq Y$ such that if $\alpha$ and $\beta$ are distinct points of $P$ then $\langle\alpha, \beta\rangle \notin R$.

Applying this theorem to $\approx$ and $\{0,1\}^{\mathbb{N}}$ we get a perfect set of mutually $\approx$ nonequivalent elements of $\{0,1\}^{\mathbb{N}}$. The proof of Mycielski's conjecture is complete.

A slight modification of the above proof gives the following theorem.

Theorem. Let $\kappa<2^{\aleph_{0}}$ be an infinite cardinal number. Suppose that $X$ is a path connected locally path connected metric space which is $\kappa$-Lindelöf (i.e., every open cover of $X$ has a subcover of size $\leq \kappa$ ). Then the power of the fundamental group of $X$ is either $\leq \kappa$ or $2^{\aleph_{0}}$.

\section{REFERENCES}

[K] K. Kuratowski, Topology, Academic Press, New York, 1966, 1968. MR 41:4467; MR 36:840 [M] J. Mycielski, Independent sets in topological algebras, Fund. Math. 55 (1964), 139-147. MR 30:3855 
[O] J. C. Oxtoby, Measure and Category, Springer-Verlag, Berlin, 1971. MR 52:14213

[S] S. Shelah, Can the fundamental group of a nice space be e.g. the rationals, Abstracts Amer.Math. Soc. 5 (1984), 217.

[Sp] E. H. Spanier, Algebraic Topology, McGraw-Hill Book Company, New York, 1966. MR 35:1007

Department of Mathematics, University of WrocŁaw, Pl. Grunwaldzki 2/4, 50-384 WrocŁaW, Poland

E-mail address: pawlikow@math.uni.wroc.pl 